\title{
SOLUTION OF HALLAM'S PROBLEM \\ ON THE TERMINAL COMPARISON PRINCIPLE FOR ORDINARY DIFFERENTIAL INEQUALITIES $\left({ }^{1}\right)$
}

BY

\section{GIOVANNI VIDOSSICH}

\begin{abstract}
We solve affirmatively the open problem raised by Hallam [3] and we apply this result to classical differential inequalities as well as to get existence and uniqueness theorems for the terminal value problem for ordinary differential equations.
\end{abstract}

Hallam in [3] points out that a comparison principle on terminal values for ordinary differential inequalities stated by Mamedov [8] has wrong proof and, in establishing a weaker form of it, raises the problem of proving Mamedov's theorem in full generality. The aim of this paper is to answer affirmatively this question. The comparison theorem proved here is exactly the analogue for terminal values of the classical comparison theorem for initial values and therefore it is even more general than Mamedov's statement.

This result is applied to improve some well-known propositions on differential inequalities for initial values as well as to prove some uniqueness and existence theorems for the terminal value problem for ordinary differential equations. In particular, an analogue of the Peano-Kneser theorem is proved: the set of solutions of terminal value problems is connected under conditions like those of Brauer [1] and Ladas and Lakshmikantham [6].

The author is grateful to T. G. Hallam for some useful comments on the preprint.

1. The general terminal comparison theorem. For $b=+\infty$ Theorem 1 below solves the problem explained in the introduction of the paper, while for $b<$ $+\infty$ Theorem 1 improves Proposition A of Cafiero [2, p. 146] since no kind of boundedness is assumed on $\omega$ and the domain of $\omega$ is more general. The proof

Received by the editors July 10, 1974 and, in revised form, March 5, 1975.

AMS (MOS) subject classifications (1970). Primary 34A40, 34A10, 34E99; Secondary 34A15, 34A45.

Key words and phrases. Differential inequalities, comparison principle, initial and terminal value problem, uniqueness and existence of solutions, connectedness of the set of solutions, maximal solution, convergence of sequences of solutions.

(1) Part of this research has been made at the "Universidade Federal de Goiás, Goiânia", to which the author is grateful for the pleasant hospitality. 
of Cafiero's result cannot be carried over in the case $b=+\infty$ since Cafiero's argument requires the solvability of the terminal value problem

$$
u^{\prime}=\bar{\omega}(t, u), \quad \lim _{t \uparrow b} u(t)=u_{\infty},
$$

for arbitrary $u_{\infty} \in \mathbf{R}, \bar{\omega}$ being a suitable deformation of $\omega$, a fact which has no guarantee in general since the continuity does not imply existence as shown by the example $\bar{\omega}(t, u)=-e^{t} \cdot u$ for $u \geqslant 0$ and $\bar{\omega}(t, u)=0$ for $u<0, u_{\infty}=1$. Note that Theorem 1 is the exact analogue for terminal values of the classical comparison theorem for initial values: only the continuity is assumed on $\omega$, any hypothesis on uniqueness and existence in the large for the Cauchy problem is completely avoided, the domain of $\omega$ is not assumed to be open in $\mathbf{R}^{2}$ nor of the form $[a, b[\times \mathbf{R}$, and $D^{*}$ is an arbitrary Dini derivative-in contrast to the assumptions of Hallam [3, Theorem 3] and Mamedov [8, Theorem 1] which are less general.

Concerning the definition of maximal solution of a terminal value problem, there are two possibilities. One is to use the "dual" of the definition of maximal solution for a Cauchy problem as it appears in Hartman [4]:

DEFINITION 1. The maximal solution in $[a, b$ [ of the terminal value problem

$$
u^{\prime}=\omega(t, u), \quad \lim _{t \uparrow b} u(t)=u_{\infty},
$$

is $a$ solution $u$ in $[a, b$ [ such that for every solution $v$ defined in an interval $[\alpha, b[\subseteq[a, b[$, we have $v \leqslant u$ in $[\alpha, b[$.

The other one is a stronger version used by Hallam [3]:

DEFINITION 2. The maximal solution in $[a, b[$ of the terminal value problem

$$
u^{\prime}=\omega(t, u), \quad \lim _{t \uparrow b} u(t)=u_{\infty},
$$

is a solution $u$ in $[a, b$ [ which is $\geqslant$ any other solution in the same interval $[a, b[$.

Obviously, a maximal solution according to Definition 1 is also the maximal solution according to Definition 2. For this reason, added to the fact that we have to solve Hallam's problem and also by the feeling that the existence of a maximal solution in the sense of Definition 2 is more probable than in the sense of Definition 1 (as supported by the proofs of Brauer [1, Theorem 5] and of Ladas and Lakshmikantham [6, Theorem 3.1]) we shall use the concept of maximal solution according to Definition 2. A necessary and sufficient condition for the existence of the maximal solution of the terminal values problems under Carathéodory hypotheses is given by the corollary of Theorem 1 of Vidossich [11], while a sufficient condition under different assumptions is given by Theorem 1 of Hallam [3] (note that the proof of [3, Theorem 2] is incomplete 
since the existence of $r_{m}, r_{M}$ is not sufficiently certified).

THEOREM 1. Let I be any right-open interval of $\mathbf{R}$ and $a, u_{\infty} \in \mathbf{R}, b \in$ $[a,+\infty]$. Let $\omega:[a, b[\times I \rightarrow \mathbf{R}$ and $v:[a, b[\rightarrow I$ be continuous, and let $u$ be the maximal solution on $[a, b[$ of the terminal value problem

$$
u^{\prime}=\omega(t, u), \quad \lim _{t \uparrow b} u(t)=u_{\infty},
$$

according to Definition 2. If $\lim _{t \uparrow b} v(t) \leqslant u_{\infty}$ and if at least one of the following conditions holds,

(i) $v$ is locally absolutely continuous on $\left[a, b\left[\right.\right.$ and $v^{\prime} \geqslant \omega(t, v)(a . e$. on $[a, b[) ;$ or

(ii) there exists a fixed Dini derivative $D^{*}$ such that $D^{*} v(t) \geqslant \omega(t, v(t))$ for all but a countable number of $t \in[a, b[$; then $v \leqslant u$ in $[a, b[$.

Of course, $v$ is locally absolutely continuous means that each $t_{0} \in[a, b[$ has a neighborhood $V$ such that $v$ is absolutely continuous in $V \cap[a, b[$. This condition is not equivalent in general to the absolute continuity of $v$ in $[a, b[$ as shown by the following example: $\left[a, b\left[=\left[0,+\infty\left[\right.\right.\right.\right.$ and $v: \mathbf{R}^{+} \rightarrow \mathbf{R}^{+}$defined by

$$
v(t)=\sum_{i=1}^{n-1} i(i+1)+n(n+1)(t-n) \quad(n \leqslant t \leqslant n+1 ; n \in \mathrm{N}) .
$$

Proof of Theorem 1. Suppose there exists $t_{0} \in\left[a, b\left[\right.\right.$ such that $v\left(t_{0}\right)>$ $u\left(t_{0}\right)$, and argue for a contradiction. Since the proof is somewhat involved, it seems convenient to summarize the basic lines. The contradiction will be achieved by showing the existence of a solution $w$ of $w^{\prime}=\omega(t, w)$ in $[a, b[$ such that $w\left(t_{0}\right)>u\left(t_{0}\right)$ and $\lim _{t \uparrow b} w(t)=u_{\infty}$. First we will construct a solution $w$ of $w^{\prime}=\omega(t, w)$ in $\left[a, t_{0}\right]$ such that $\left.w\left(t_{0}\right) \in\right] u\left(t_{0}\right), v\left(t_{0}\right)$ [. This could be done simply by using the general convergence theorem for solutions of Cauchy problems, Hartman [4, Chapter II, Theorem 3.2], if $x^{\prime}=\omega(t, x)$ has uniqueness of the Cauchy problem. But since we do not have such a uniqueness, we will get the desired result by combining some ideas involved in the proof of the mentioned convergence theorem with the connectedness of the set of solutions of a Cauchy problem. Then we will extend conveniently $w$ to the right of $t_{0}$ by using locally a differential inequalities technique. To achieve this goal, we need the strict inequality $D^{*} v>\omega(t, v)$ instead of $D^{*} v \geqslant \omega(t, v)$. Therefore $\omega$ will be deformed in a suitable $\omega_{n}$ with $\lim _{n} \omega_{n}=\omega$. We note also we shall use repeatedly the observation that if a solution $x$ touches $u$ for a time $t$, then $x \leqslant$ $u$ in $[a, t]$-as it will be deduced from the maximality of $u$.

The first step to get a contradiction against $v\left(t_{0}\right)>u\left(t_{0}\right)$, is to prove the following statement: 
(1) There exists a solution $w$ of $w^{\prime}=\omega(t, w)$ in $\left[a, t_{0}\right]$ such that $u\left(t_{0}\right)<$ $w\left(t_{0}\right)<v\left(t_{0}\right)$.

To this aim, let $V=]-\infty$, sup $I\left[\right.$ and $g:\left[a, t_{0}\right] \times V \rightarrow \mathbf{R}$ defined by

$$
g(t, x)= \begin{cases}\omega(t, x) & \text { if } x \geqslant u(t), \\ \omega(t, u(t)) & \text { if } x<u(t) .\end{cases}
$$

Obviously $g$ is continuous and $u^{\prime}=g(t, u)$ in $\left[a, t_{0}\right]$. Let $m_{1}, m_{2}$ be the minimum and maximum of $u$ on $\left[a, t_{0}\right]$, let $\epsilon>0$ be such that $m_{2}+2 \epsilon<\sup I$, let $M$ be the maximum of $|g|$ on the compact set $\left[a, t_{0}\right] \times\left[m_{1}-2 \epsilon, m_{2}+2 \epsilon\right]$, and let $\left.\delta \in] 0, t_{0}-a\right]$ be so small that $\delta M<\epsilon$. Let $A$ be the set of all $t \in$ ] $\left.a, t_{0}\right]$ such that there exists a sequence $\left(x_{n}\right)_{n}$ of solutions in $[a, t]$ of $x^{\prime}=$ $g(t, x)$ such that

$$
u \leqslant x_{n} \leqslant m_{2}+2 \epsilon \text { in }[a, t] \text { and } u(t)<x_{n}(t) \quad\left(n \in \mathbf{Z}^{+}\right),
$$

and

$$
\lim _{n} x_{n}(t)=u(t)
$$

We claim $a+\delta \in A$. Let us first establish the following statement.

(2) For every $x_{0} \in[u(a), u(a)+\epsilon]$, all solutions $x$ of the Cauchy problem $x^{\prime}=g(t, x), x(a)=x_{0}$ exist in $[a, a+\delta]$ and satisfy $x \leqslant m_{2}+2 \epsilon$ and moreover, if $x_{0}>u(a), u<x$.

Choose $x_{0}$, and let $x$ be any corresponding solution. Let $D$ be the maximal interval of existence of $x$. Define

$$
\eta=\sup \left\{t \in D \cap[a, a+\delta] \mid m_{1}-2 \epsilon \leqslant x \leqslant m_{2}+2 \epsilon \text { in }[a, t]\right\} .
$$

If $\eta<a+\delta$, then we have

$$
\left|x(\eta)-x_{0}\right| \leqslant \int_{a}^{\eta}|g(s, x(s))| d s \leqslant \int_{a}^{\eta} M d s<\epsilon,
$$

and therefore

$$
m_{1}-2 \epsilon<x_{0}-\epsilon<x(\eta)<x_{0}+\epsilon<m_{2}+2 \epsilon .
$$

By the continuity of $x$, there is $\left.\left.\eta_{0} \in\right] \eta, a+\delta\right]$ such that

$$
m_{1}-2 \epsilon<x(t)<m_{2}+2 \epsilon \quad\left(\eta \leqslant t \leqslant \eta_{0}\right),
$$

and we have a contradiction against the definition of $\eta$. Therefore $\eta=a+\delta$, so that $x$ exists on $[a, a+\delta]$ and $x \leqslant m_{2}+2 \epsilon$. If $u<x$ does not hold for $x_{0}>$ $u(a)$, then there exists a $t_{1} \in[a, a+\delta]$ such that $u\left(t_{1}\right)=x\left(t_{1}\right)$ and $u<x$ in $\left[a, t_{1}[\right.$. Then the function $y:[a, b[\rightarrow \mathbf{R}$ defined by 


$$
y(t)= \begin{cases}x(t) & \text { for } t \leqslant t_{1}, \\ u(t) & \text { for } t>t_{1},\end{cases}
$$

is a solution of our terminal value problem (since $y^{\prime}=\omega(t, y)$ in $\left[a, t_{1}\right]$ and $\left.\lim _{t \uparrow t_{1}} y^{\prime}(t)=\omega\left(t_{1}, y\left(t_{1}\right)\right)=\lim _{t \downarrow t_{1}} y^{\prime}(t)\right)$, so that $y \leqslant u$ by the maximality of $u$. This yields a contradiction against $u(a)<x(a)$. In conclusion, (2) holds.

Now we distinguish two cases:

Case 1. $u$ is the maximal solution of the Cauchy problem

$$
x^{\prime}=g(t, x), \quad x(a)=u(a),
$$

in $[a, a+\delta]$. Let $x_{n, 0}$ be a sequence in $] u(a), u(a)+\epsilon$ [ converging to $u(a)$. By (2), there is a solution $x_{n}$ in $[a, a+\delta]$ of the Cauchy problem

$$
x_{n}^{\prime}=g\left(t, x_{n}\right), \quad x_{n}(a)=x_{n, 0},
$$

such that $u<x_{n} \leqslant m_{2}+2 \epsilon$ in $[a, a+\delta]$. By Ascoli's theorem, there is a subsequence $\left(x_{n_{k}}\right)_{k}$ converging uniformly in $[a, a+\delta]$ to a solution $x$ of (3). Since $u$ is the maximal solution of (3), $x \leqslant u$. Since $x \geqslant u$ by $u<x_{n_{k}}$, we have $u=$ $x$ and hence with $\left(x_{n_{k}}\right)_{k}$ the condition for $a+\delta \in A$ is satisfied.

Case 2. $u$ is not the maximal solution of (3). By (2), all solutions of (3), hence in particular the maximal solution $u_{M}$, exist in $[a, a+\delta]$. Since $u_{M} \neq u$ and $u_{M} \geqslant u$ in $[a, a+\delta]$, we have $u_{M}(a+\delta)>u(a+\delta)$ : for, otherwise the function $y_{1}:[a, b[\rightarrow \mathbf{R}$ defined by

$$
y_{1}(t)= \begin{cases}u_{M}(t) & \text { for } t \leqslant a+\delta, \\ u(t) & \text { for } t>a+\delta,\end{cases}
$$

would be a solution of our terminal value problem, and so we would have $y_{1} \leqslant$ $u$, which yields the contradiction $u_{M} \leqslant u$. By the Peano-Kneser theorem, the values at $a+\delta$ of all solutions of (3) form a connected set, hence an interval. Therefore, because $u_{M}(a+\delta)>u(a+\delta)$, there is a sequence $\left(x_{n}\right)_{n}$ of solutions of (3) in $[a, a+\delta]$ such that $x_{n}(a+\delta)>u(a+\delta)$ for all $n$ and $\lim _{n} x_{n}(a+\delta)=$ $u(a+\delta)$. By (2), $x_{n} \leqslant m_{2}+2 \epsilon$ in $[a, a+\delta]$. If $x_{n} \ngtr u$, then let $s_{n}=$ $\inf \left\{t \in[a, a+\delta] \mid u<x_{n}\right.$ in $\left.[t, a+\delta]\right\}$ and define $\bar{x}_{n}:[a, a+\delta] \rightarrow \mathbf{R}$ by

$$
\bar{x}_{n}(t)= \begin{cases}u(t) & \text { for } t<s_{n}, \\ x_{n}(t) & \text { for } t \geqslant s_{n} .\end{cases}
$$

Obviously $\bar{x}_{n}$ is a solution of (3) such that $\bar{x}_{n} \geqslant u$. Therefore with $x_{n}^{*}=x_{n}$ or $\bar{x}_{n}$ the condition for $a+\delta \in A$ is satisfied.

Thus $a+\delta \in A$. Define $\alpha=\sup A$ and argue to show $\alpha \in A$ and $\alpha=t_{0}$. Let 
$\left.\left.t_{2} \in\right] \alpha-\delta, \alpha\right] \cap A$ and let $\left(x_{n}\right)_{n}$ be the sequence associated to $t_{2}$ by the condition $t_{2} \in A$. There is an $n_{0}$ such that $\left.x_{n}\left(t_{2}\right) \in\right] u\left(t_{2}\right), u\left(t_{2}\right)+\epsilon\left[\right.$ for $n \geqslant n_{0}$. Repeating the argument used to prove (2), we have that each $x_{n}$ can be extended in $J=\left[t_{2}, t_{2}+\delta\right] \cap\left[a, t_{0}\right]$ as a solution of $x^{\prime}=g(t, x)$, which we shall still denote by $x_{n}$, and which satisfies there $u<x_{n} \leqslant m_{2}+2 \epsilon$. Let $\beta$ be any point of $\left[t_{2}, \sup J\right]$. By Ascoli's theorem, there is a subsequence $\left(x_{n_{k}}\right)_{k}$ of $\left(x_{n}\right)_{n}$ converging uniformly in $[a, \beta]$ to a solution $\bar{x}$ of $x^{\prime}=g(t, x)$. The following two cases can occur:

Case I. $\bar{x}(\beta)=u(\beta)$. Then with $\left(x_{n_{k}}\right)_{k}$ the condition for $\beta \in A$ is satisfied.

Case II. $\bar{x}(\beta)>u(\beta)$. Then there is a sequence $\left(y_{n, 0}\right)_{n}$ in $] u(\beta), \bar{x}(\beta)[$ converging to $u(\beta)$. Let $y_{n}$ be a solution of the Cauchy problem

$$
y_{n}^{\prime}=g\left(t, y_{n}\right), \quad y_{n}(\beta)=y_{n, 0},
$$

and let $I_{n} \subseteq[a, \beta]$ its maximal interval of existence at the left of $\beta$ (hence, we should have spoken of the terminal value problem). Once more, we distinguish two cases:

Subcase 1. There exists $n_{0}$ such that $y_{n}>u$ in $I_{n}$ for all $n \geqslant n_{0}$. If there is $n_{1} \geqslant n_{0}$ such that $y_{n}<\bar{x}$ in $I_{n}$ for all $n \geqslant n_{1}$, then every $y_{n}, n \geqslant n_{1}$, is bounded where it exists and moreover $\overline{y_{n}\left(I_{n}\right)} \subseteq V$ : therefore well-known facts imply that $I_{n}=[a, \beta]$. If such an $n_{1}$ does not exist, then there is $n_{k} \rightarrow \infty$ such that $y_{n_{k}}$ touches $\bar{x}$ in some point: let $\xi_{k}=\sup \left\{t \in I_{n_{k}} \mid y_{n_{k}}(t)=\bar{x}(t)\right\}$. Then we define

$$
z_{k}(t)= \begin{cases}\bar{x}(t) & \text { for } t \in\left[a, \xi_{k}\right], \\ y_{n_{k}}(t) & \text { for } t \in\left[\xi_{k}, \beta\right],\end{cases}
$$

and with $\left(y_{n}\right)_{n \geqslant n_{1}}$ or $\left(z_{k}\right)_{k}$ we have the condition for $\beta \in A$.

Subcase 2. There exists $n_{k} \rightarrow \infty$ such that $y_{n_{k}} \ngtr u$. Then let $\zeta_{k}=$ $\sup \left\{t \in I_{n_{k}} \mid y_{n_{k}}(t)=u(t)\right\}$. There are still two possibilities: there exists $k_{0}$ such that $y_{n_{k}}<\bar{x}$ in $\left[\zeta_{k}, \beta\right]$ for $k \geqslant k_{0}$, or there exists $k_{i} \rightarrow \infty$ such that $y_{n_{k_{i}}}$ touches $\bar{x}$ in $\left[\zeta_{k}, \beta\right]$. If $y_{n_{k}}<\bar{x}$ in $\left[\zeta_{k}, \beta\right]$ for $k \geqslant k_{0}$, then defining

$$
z_{k}(t)= \begin{cases}u(t) & \text { for } t \in\left[a, \zeta_{k}\right], \\ y_{n_{k}}(t) & \text { for } t \in\left[\zeta_{k}, \beta\right],\end{cases}
$$

we have the condition for $\beta \in A$. If there exists $k_{i} \rightarrow \infty$ such that $y_{n_{k_{i}}}$ touches $\bar{x}$ in $\left[\zeta_{k}, \beta\right]$, then let $\sigma_{i}=\sup \left\{t \in\left[\zeta_{k}, \beta\right] \mid y_{n_{k_{i}}}(t)=\bar{x}(t)\right\}$. Defining

$$
z_{i}(t)= \begin{cases}\bar{x}(t) & \text { for } t \in\left[a, \sigma_{i}\right], \\ y_{n_{k_{i}}}(t) & \text { for } t \in\left[\sigma_{i}, \beta\right],\end{cases}
$$

we have the condition for $\beta \in A$. 
Therefore, $\beta \in A$. For $\beta=\alpha$ we have $\alpha \in A$. If $\alpha<t_{0}$, then for $\beta=\sup J$ we would have a contradiction against $\alpha=\sup A$. Therefore $\alpha=t_{0}$. By taking $n$ sufficiently large in the sequence characterizing $t_{0} \in A$, we have the existence of a solution $w$ of $x^{\prime}=g(t, x)$ in $\left[a, t_{0}\right]$ such that $u\left(t_{0}\right)<w\left(t_{0}\right)<v\left(t_{0}\right)$ and $u$ $\leqslant w$ in $\left[a, t_{0}\right]$. Therefore $w^{\prime}=\omega(t, w)$ in $\left[a, t_{0}\right]$ and (1) holds.

Now let

$$
\gamma=\sup \left\{t \in \left[t_{0}, b\left[\mid v>u \text { in }\left[t_{0}, t\right]\right\}\right.\right.
$$

By continuity and $\lim _{t \uparrow b} v(t) \leqslant u_{\infty}$ we have

$$
\lim _{t \uparrow \gamma} v(t)=\lim _{t \uparrow \gamma} u(t)
$$

For every $n \in \mathbf{Z}^{+}$define $\omega_{n}:\left[t_{0}, \gamma[\times I \rightarrow \mathbf{R}\right.$ by

$$
\omega_{n}(t, x)=\omega(t, x)+(u(t)-x) / n \text {. }
$$

We have

$$
\omega_{n}(t, v(t))<\omega(t, v(t)) \text { and } u^{\prime}(t)=\omega_{n}(t, u(t)) \quad\left(t_{0} \leqslant t<\gamma\right) .
$$

We claim

(5) For every $n \in \mathrm{Z}^{+}$there exists a solution $u_{n}$ in [ $t_{0}, \gamma$ [ of the Cauchy problem

$$
u_{n}^{\prime}=\omega_{n}\left(t, u_{n}\right), \quad u_{n}\left(t_{0}\right)=w\left(t_{0}\right)
$$

such that

$$
u(t) \leqslant u_{n}(t)<v(t) \quad\left(t_{0} \leqslant t<\gamma\right)
$$

To prove (5), fix $n \in \mathbf{Z}^{+}$. Let $w_{n}$ be a solution of the Cauchy problem

$$
w_{n}^{\prime}=\omega_{n}\left(t, w_{n}\right), \quad w_{n}\left(t_{0}\right)=w\left(t_{0}\right),
$$

and $M_{n} \subseteq\left[t_{0}, \gamma[\right.$ its maximal interval of existence. Define

$$
\alpha_{n}=\sup M_{n} \text { and } t_{n}=\sup \left\{t \in M_{n} \mid w_{n}(s)<v(s) \text { for all } s \in\left[t_{0}, t\right]\right\} .
$$

In order to prove the equality $t_{n}=\alpha_{n}$, assume $t_{n}<\alpha_{n}$. Then by continuity we have $w_{n}\left(t_{n}\right)=v\left(t_{n}\right)$ and consequently

$$
\omega_{n}\left(t_{n}, w_{n}\left(t_{n}\right)\right)=\omega_{n}\left(t_{n}, v\left(t_{n}\right)\right)<\omega\left(t_{n}, v\left(t_{n}\right)\right) .
$$

The continuity of $\omega_{n}\left(\cdot, w_{n}(\cdot)\right)$ and $\omega(\cdot, v(\cdot))$ implies the existence of an interval $I_{n}=\left[t_{n}-\delta_{n}, t_{n}\right] \subseteq\left[t_{0}, \alpha_{n}[\right.$ such that

$$
\omega_{n}\left(t, w_{n}(t)\right)<\omega(t, v(t)) \quad\left(t \in I_{n}\right)
$$


and hence

$$
w_{n}(t)-w_{n}(s)=\int_{s}^{t} \omega_{n}\left(\xi, w_{n}(\xi)\right) d \xi<\int_{s}^{t} \omega(\xi, v(\xi)) d \xi \quad\left(s, t \in I_{n} ; s<t\right)
$$

If condition (i) of the hypothesis holds, we take $I_{n}$ so small that $v$ is absolutely continuous on $I_{n}$. Then $v(t)-v(s)=\int_{s}^{t} v^{\prime}(\xi) d \xi$ for $s, t \in I_{n}$ and so from (6) and $v^{\prime} \geqslant \omega(t, v)$ we get $w_{n}\left(t_{n}\right)-w_{n}\left(t_{n}-\delta_{n}\right)<v\left(t_{n}\right)-v\left(t_{n}-\delta_{n}\right)$, which implies $w_{n}\left(t_{n}-\delta_{n}\right)>v\left(t_{n}-\delta_{n}\right)$, a contradiction. If (ii) holds, put

$$
V_{n}(t)=v\left(t_{n}-\delta_{n}\right)+\int_{t_{n}-\delta_{n}}^{t} \omega(\xi, v(\xi)) d \xi \quad\left(t \in I_{n}\right)
$$

From (6) we have

$$
w_{n}(t)<V_{n}(t) \quad\left(t \in I_{n}\right)
$$

By $V_{n}^{\prime}(t) \leqslant D^{*} v$ in all of $I_{n}$ except a countable subset, we have $D^{*}\left(V_{n}-v\right) \leqslant 0$ in all of $I_{n}$ except a countable subset. Then Zygmund's lemma on Dini derivatives (cf. Lakshmikantham and Leela [7, Lemma 1.2.1]) implies that $V_{n}-v$ is decreasing in $I_{n}$. Thus from $V_{n}\left(t_{n}-\delta_{n}\right)-v\left(t_{n}-\delta_{n}\right)=0$ it follows that

$$
V_{n}(t) \leqslant v(t) \quad\left(t \in I_{n}\right)
$$

from which we derive $w_{n}\left(t_{n}\right)<V_{n}\left(t_{n}\right) \leqslant v\left(t_{n}\right)$, a contradiction. Therefore $t_{n}=$ $\alpha_{n}$. Now define

$$
s_{n}=\sup \left\{t \in M_{n} \mid u(s)<w_{n}(s) \text { for all } s \in\left[t_{0}, t\right]\right\} .
$$

If $s_{n}<\alpha_{n}$, then by continuity $u\left(s_{n}\right)=w_{n}\left(s_{n}\right)$ and so

$$
v_{n}(t)= \begin{cases}w_{n}(t) & \text { for } t_{0} \leqslant t<s_{n}, \\ u(t) & \text { for } s_{n} \leqslant t<\gamma,\end{cases}
$$

is a solution of $x^{\prime}=\omega_{n}(t, x)$ on $\left[t_{0}, \gamma\left[\right.\right.$ and $u \leqslant v_{n}<v$ on $\left[t_{0}, \gamma[\right.$. Therefore (5) holds for $u_{n}=v_{n}$ if $s_{n}<\alpha_{n}$. Now assume $s_{n}=\alpha_{n}$. If $\alpha_{n}=\gamma$, then (5) holds for $u_{n}=w_{n}$. If $\alpha_{n}<\gamma$, then $\lim _{t \uparrow \alpha_{n}} w_{n}(t)$ exists since the boundedness of $w_{n}$ in $\left[t_{0}, s_{n}\left[=\left[t_{0}, \alpha_{n}\right.\right.\right.$ [ implies that $w_{n}$ is Lipschitzean. In view of the maximality of $M_{n}$ and $\lim _{t \uparrow \alpha_{n}} w_{n}(t) \in\left[u\left(\alpha_{n}\right), v\left(\alpha_{n}\right)\right] \subseteq I, w_{n}$ must be defined at $\alpha_{n}$ as a solution of $x^{\prime}=\omega_{n}(t, x)$. We claim $u\left(\alpha_{n}\right)=w_{n}\left(\alpha_{n}\right)$. If $u\left(\alpha_{n}\right)<$ $w_{n}\left(\alpha_{n}\right)$, then $w_{n}\left(\alpha_{n}\right)$ is an interior point of $I$ since $I$ is a right-open interval. Therefore Peano's existence theorem is applicable to the Cauchy problem

$$
x^{\prime}=\omega_{n}(t, x), \quad x\left(\alpha_{n}\right)=w_{n}\left(\alpha_{n}\right),
$$

and we contradict the maximality of $M_{n}$. Therefore $u\left(\alpha_{n}\right)=w_{n}\left(\alpha_{n}\right)$. Then we define the function $v_{n}$ as in (7), which is possible since $s_{n}=\alpha_{n}$ in the present 
assumptions, and so (5) holds for $u_{n}=v_{n}$. In conclusion, (5) is true.

Now, let $\left(\beta_{k}\right)_{k}$ be a sequence in $\left[t_{0}, \gamma[\right.$ converging to $\gamma$. By (5), $\left(\left.u_{n}\right|_{\left[t_{0}, \beta_{k}\right]}\right)_{n}$ is an equicontinuous and equibounded sequence for all $k$. Therefore by Ascoli's theorem there is a subsequence $\left(u_{k_{1, n}}\right)_{n}$ of $\left(u_{n}\right)_{n}$ converging uniformly on $\left[t_{0}, \beta_{1}\right]$ such that $k_{1, n}<k_{1, n+1}$ for all $n$, there is a subsequence $\left(u_{k_{2, n}, n}\right)_{n}$ of $\left(u_{k_{1, n}}\right)_{n}$ converging uniformly on $\left[t_{0}, \beta_{2}\right]$ such that $k_{2, n}<k_{2, n+1}$ for all $n$, and so on for $i=3, \ldots, \infty$. Then $\left(u_{k_{n, n}}\right)_{n}$ is a subsequence of $\left(u_{n}\right)_{n}$ converging uniformly on compact intervals of $\left[t_{0}, \gamma\left[\right.\right.$ to a function $w_{0}$. By this and $\lim _{n} \omega_{n}=\omega$ uniformly on bounded subsets of $[a, b[\times I$, it is easy to deduce from Riemann's convergence theorem and from

$$
u_{k_{n, n}}(t)=w\left(t_{0}\right)+\int_{t_{0}}^{t}\left(\omega_{n}\left(s, u_{k_{n, n}}(s)\right) \pm \omega\left(s, u_{k_{n, n}}(s)\right)\right) d s
$$

that

$$
w_{0}(t)=w\left(t_{0}\right)+\int_{t_{0}}^{t} \omega\left(s, w_{0}(s)\right) d s \quad\left(t_{0} \leqslant t \leqslant \beta_{i}\right)
$$

for all $i \in \mathbf{Z}^{+}$. Therefore $w_{0}^{\prime}=\omega\left(t, w_{0}\right)$ in $\left[t_{0}, \gamma[\right.$. From (5) and (4) it follows that

$$
\lim _{t \uparrow \gamma} w_{0}(t)=\lim _{t \uparrow \gamma} u(t)
$$

If $\boldsymbol{\gamma}=b$, then the function $\bar{w}:[a, b[\rightarrow \mathbf{R}$ defined by

$$
\bar{w}(t)= \begin{cases}w(t) & \text { for } t \in\left[a, t_{0}\right], \\ w_{0}(t) & \text { for } t \in\left[t_{0}, b[,\right.\end{cases}
$$

is a solution of our terminal value problem and therefore $\bar{w} \leqslant u$ by the maximality of $u$. This contradicts $\bar{w}\left(t_{0}\right)>u\left(t_{0}\right)$. If $\gamma<b$, then we define $\bar{w}:[a, b[\rightarrow \mathbf{R}$ by

$$
\bar{w}(t)= \begin{cases}w(t) & \text { for } t \in\left[a, t_{0}\right], \\ w_{0}(t) & \text { for } t \in\left[t_{0}, \gamma[,\right. \\ u(t) & \text { for } t \in[\gamma, b[\end{cases}
$$

and we have the same contradiction. Q.E.D.

2. Applications to classical differential inequalities. The following result improves the well-known comparison theorem for initial values since $\left(a, u_{\infty}\right)$ is assumed to be only in the closure of the domain of $\omega$. Note that Theorem 2 works with the concept of maximal solution of a Cauchy problem "dual" to that of Definition 2.

THEOREM 2. Let $a<b$ and $u_{\infty}$ be real numbers, $I \subseteq \mathbf{R}$ a right-open inter- 
val and $\omega:] a, b] \times I \rightarrow \mathbf{R}$ continuous. Let $v:] a, b] \rightarrow I$ be continuous and $u$ the maximal solution of

$$
u^{\prime}=\omega(t, u), \quad \lim _{t \downarrow a} u(t)=u_{\infty} .
$$

If $\lim _{t \downarrow a} v(t) \leqslant u_{\infty}$ and either $v$ is absolutely continuous on compact intervals of ]$a, b]$ and

$$
\left.\left.v^{\prime} \leqslant \omega(t, v) \quad(\text { a.e. in }] a, b\right]\right),
$$

or $D^{*} v \leqslant \omega(t, v)$ in $\left.] a, b\right]$ except a countable subset, $D^{*}$ being any fixed Dini derivative, then $v \leqslant u$ in $] a, b]$.

Proof. Define $g:[1 /(b-a),+\infty[\times I \rightarrow \mathbf{R}$ by

$$
g(t, x)=-\left(1 / t^{2}\right) \omega(a+1 / t, x) .
$$

Obviously if $x^{\prime}=\omega(t, x)$ then $\bar{x}(t)=x(a+1 / t), t \geqslant 1 /(b-a)$, is a solution of $y^{\prime}=g(t, y)$, while if $y^{\prime}=g(t, y)$ then $x(t)=y(1 /(t-a)), b \geqslant t>a$, is a solution of $x^{\prime}=\omega(t, x)$. Therefore $\bar{u}$ is the maximal solution of the terminal value problem

$$
y^{\prime}=g(t, y), \quad \lim _{t \uparrow \infty} y(t)=u_{\infty} .
$$

The function $\bar{v}(t)=v(a+1 / t)$ is absolutely continuous on compact intervals when $v$ is, since $t \rightarrow a+1 / t$ is Lipschitz on compact intervals (being continuously differentiable). Therefore we are in position to apply Theorem 1 to $\bar{u}, \bar{v}$ and $g$ in order to get $\bar{v} \leqslant \bar{u}$ in $[1 /(b-a),+\infty$ [. Therefore $v \leqslant u$ in $] a, b]$. Q.E.D.

The following result improves Lakshmikantham and Leela [7, Theorem 1.2.1] since the inequalities $D^{*} v \geqslant \omega(t, v)$ is not assumed everywhere and since $D^{*}$ is an arbitrary Dini derivative.

Theorem 3. Let $I \subseteq \mathbf{R}$ be a right-open interval and $\omega:[a, b] \times I \rightarrow \mathbf{R}$ continuous such that the Cauchy problem

$$
x^{\prime}=\omega(t, x), \quad x\left(t_{0}\right)=u_{0},
$$

has at most one solution at the left of $t_{0}$ for all $\left(t_{0}, u_{0}\right)$. If $v:[a, b] \rightarrow I$ is absolutely continuous and

$$
\left.v^{\prime} \geqslant \omega(t, v) \quad \text { (a.e. in }[a, b]\right),
$$

or $v$ is continuous and $D^{*} v \geqslant \omega(t, v)$ for all but a countable number of $t, D^{*}$ being any fixed Dini derivative, then

$$
u^{\prime}=\omega(t, u) \text { in }[a, b] \text { and } u(a)<v(a)
$$

implies $u<v$ in $[a, b]$. 
Proof. Let $A=\{t \in[a, b] \mid u<v$ in $[a, t]\}$ and $\beta=\sup A$. If $\beta<b$, then $u(\beta)=v(\beta)$ by continuity and the hypotheses. Since $x^{\prime}=\omega(t, x), x(\beta)=$ $u(\beta)$ has a unique solution at the left of $\beta, u$ is the maximal solution of the terminal value problem

$$
x^{\prime}=\omega(t, x), \quad \lim _{t \uparrow \beta} x(t)=u(\beta)
$$

(note that any solution of this terminal value problem satisfies $x^{\prime}(\beta)=\omega(\beta, u(\beta)$ ) since $\left.\lim _{t \uparrow \beta} x^{\prime}(t)=\omega(\beta, u(\beta))\right)$. Therefore, by Theorem $1, v \leqslant u$ in $[a, \beta[$, which is impossible since $u(a)<v(a)$. Therefore $\beta=b$. If $u(b)=v(b)$, then by the same argument we get a contradiction against $u(a)<v(a)$. Thus $b \in A$. Q.E.D.

3. Applications to uniqueness of the terminal value problem for ordinary differential equations. In this section we use Theorem 1 to get some result about uniqueness of solutions of the terminal value problem. In our approach to uniqueness there is implicit a new comparison viewpoint which has been explored in Vidossich [10].

Theorem 4. Let $X$ be a Banach space, $A \subseteq X, a \in \mathbf{R}, b \in[a,+\infty], f$ : $[a, b[\times A \rightarrow X$ bounded on bounded sets and $\omega:[a, b[\times[0, \beta[\rightarrow \mathbf{R}$ continuous. If

(i) $(f(t, x)-f(t, y), x-y)_{-} \geqslant \omega(t,\|x-y\|)\|x-y\|$ for all $t, x, y$; and

(ii) $u^{\prime}=\omega(t, u), \lim _{t \uparrow b} u(t)=0$ has $u \equiv 0$ as maximal solution;

then for every $x_{\infty} \in X$ the terminal value problem

$$
x^{\prime}=f(t, x), \quad \lim _{t \uparrow b} x(t)=x_{\infty},
$$

has at most one weak solution which is bounded on compact sets.

Recall that weak solution of $x^{\prime}=f(t, x)$ means a function $x:[a, b[\rightarrow X$ such that for every $t$ we have

$$
d h(x(t)) / d t=h(f(t, x(t))) \quad\left(h \in X^{*}\right) .
$$

Of course, when $X=\mathrm{R}^{n}$ the weak solutions coincide with the strong solutions (since then $h$ runs in particular in the set of canonical projections $\mathbf{R}^{n} \rightarrow \mathbf{R}$ ).

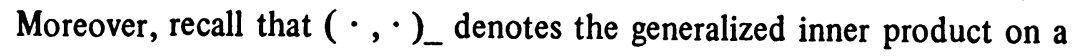
Banach space $X$ :

$$
(x, y)_{-}=\inf \{h(x) \mid h \in J(y)\}
$$

where $J: X \rightarrow 2^{X^{*}}$ is the duality map:

$$
J(x)=\left\{h \in X^{*} \mid\|h\|=\|x\|, h(x)=\|x\|^{2}\right\} .
$$


When $X$ is a Hilbert space, like $\mathrm{R}^{n}$ for example, then $(\cdot, \cdot)_{-}$coincides with the inner product.

PROOF OF THEOREM 4. Let $x, y$ be two weak solutions, bounded on compact sets, of our terminal value problem. Put $z=x-y$. Since $f$ is bounded on bounded sets, $\|z(\cdot)\|$ is Lipschitz, hence absolutely continuous, on compact intervals. $\left({ }^{1}\right)$ Therefore by Kato $[5$, Lemma 1.31, the function $v(t)=\|z(t)\|^{2}$ satisfies a.e. the condition

$$
\begin{aligned}
v^{\prime}(t) & =2(f(t, x(t))-f(t, y(t)), x(t)-y(t))_{-} \\
& \geqslant 2 \omega(t,\|z(t)\|)\|z(t)\|=2 \omega(t, \sqrt{v}) \sqrt{v} .
\end{aligned}
$$

It is easily seen that if $u \geqslant 0$ is the maximal solution of $u^{\prime}=\omega(t, u), \lim _{t \uparrow b} u(t)=0$, then $u^{2}$ is the maximal solution of

$$
w^{\prime}=g(t, w), \quad \lim _{t \uparrow b} w(t)=0,
$$

where $g(t, w)=2 \omega(t, \sqrt{w}) \sqrt{w}, w \geqslant 0$. Therefore from Theorem 1 we have $v \leqslant$ 0 in $[a, b[$, i.e. $x=y$. Q.E.D.

Corollary 1. Let $f, \omega$ be as in Theorem 4. If at least one of the following conditions:

(i)* $\left|(f(t, x)-f(t, y), x-y) \_\right| \leqslant \omega(t,\|x-y\|)\|x-y\| ;$ or

(i) $* * \quad\|f(t, x)-f(t, y)\| \leqslant \omega(t,\|x-y\|)$;

holds, and if $u^{\prime}=-\omega(t, u), \lim _{t \uparrow b} u(t)=0$ has $u \equiv 0$ as maximal solution, then for every $x_{\infty} \in X$ there is at most one weak solution of $x^{\prime}=f(t, x), \lim _{t \uparrow b} x(t)$ $=x_{\infty}$ which is bounded on bounded sets.

Proof. The conclusion of the theorem for hypothesis (i)* is a direct consequence of Theorem 4 since (i)* implies

$$
(f(t, x)-f(t, y), x-y)_{-} \geqslant-\omega(t,\|x-y\|)\|x-y\|,
$$

while (i) ${ }^{* *}$ is a special case of $(\mathrm{i})^{*}$ since $\left|(x, y) \_\right| \leqslant\|x\|\|y\|$. Q.E.D.

The interest of the following corollary lies in its providing a possible choice for the comparison term $\omega$ in the two preceding results: the $\omega$ of Corollary 1 can be decreasing in the second variable, while the $\omega$ of Theorem 4 can be increasing in the second variable.

Corollary 2. Let $I \subseteq \mathbf{R}$ and let $\omega:[a, b[\times I \rightarrow \mathbf{R}$ be continuous with $\omega(t, \cdot)$ increasing for every $t$. Then the terminal value problem

$$
u^{\prime}=\omega(t, u), \quad \lim _{t \uparrow b} u(t)=u_{\infty},
$$

(1) For every $t, s$ there is $h \in X^{*}$ such that $\|h\|=1$ and $h(z(t)-z(s))=\|z(t)-z(s)\|$. Therefore from the mean value theorem applied to $h \circ z$ we have $\|z(t)-z(s)\|=\ln (z(t))-$ $h(z(s))|<| t-\operatorname{sisup}_{\xi} \mid h\left(f(\xi, x(\xi))-h\left(f(\xi, x(\xi))|<| t-s \mid \sup _{\xi}\|f(\xi, x(\xi))-f(\xi, x(\xi))\|\right.\right.$. 
has at most one solution for every $u_{\infty} \in \mathbf{R}$.

Proof. Since $(\omega(t, u)-\omega(t, v))(u-v) \geqslant 0$ and the inner product of $\mathbf{R}$ coincides with the ordinary product, we have only to apply Theorem 4 with $f=$ $\omega$ and with the new $\omega \equiv 0$. Q.E.D.

4. Applications to the existence of solutions of terminal value problems for ordinary differential equations. The aim of this section is to deduce from Theorem 1 some existence theorems for terminal value problems. We are interested also in showing that the set of solutions is connected. To this aim we shall define the analogue for terminal values of the well-known Tonnelli approximants for the initial value problem.

TheOREM 5. Let $f:\left[a,+\infty\left[\times \mathbf{R}^{n} \rightarrow \mathbf{R}^{n}\right.\right.$ and $\omega:\left[a,+\infty\left[\times \mathbf{R}^{+} \rightarrow \mathbf{R}\right.\right.$ be continuous such that

$$
(f(t, x) \mid x) \geqslant \omega(t,\|x\|)\|x\| \quad(\text { all } t, x) .
$$

If for every $\epsilon>0$ there is $h_{\epsilon} \in L_{\text {loc }}^{1}\left(\left[a,+\infty D\right.\right.$ such that $\int_{a}^{+\infty} h_{\epsilon}<+\infty$ and

$$
\|f(t, x)\| \leqslant h_{\epsilon}(t) \quad(t \geqslant a ;\|x\| \leqslant \epsilon),
$$

and if for every $u_{\infty} \in \mathbf{R}^{+}$there is a maximal solution in $[a,+\infty[$ of

$$
u^{\prime}=\omega(t, u), \quad \lim _{t \uparrow \infty} u(t)=u_{\infty},
$$

then for every $x_{\infty} \in X$ there is a solution on $[a,+\infty[$ of the terminal value problem

$$
x^{\prime}=f(t, x), \quad \lim _{t \uparrow \infty} x(t)=x_{\infty},
$$

and the set of solutions is connected and compact for the topology of uniform convergence on $[a,+\infty[$.

Proof. We shall look at $[a,+\infty]$ as a compact topological space. Let $X$ be the Banach space of all continuous functions $[a,+\infty] \rightarrow \mathbf{R}^{n}$ with the sup norm $\|\cdot\|_{\infty}$. Define the operators $T, T_{m}: X \rightarrow X, m \in \mathrm{Z}^{+}$, by

$$
\begin{aligned}
& T x(t)= x_{\infty}-\int_{t}^{\infty} f(s, x(s)) d s, \\
& T_{m} x(t)=\left\{\begin{array}{l}
x_{\infty} \text { for } t \geqslant a+m, \\
x_{\infty}-\int_{t+1 / m}^{a+m} f(s, x(s)) d s+\int_{a+m+1 / m}^{a+m} f(s, x(s)) d s \\
\text { for } t<a+m .
\end{array}\right.
\end{aligned}
$$

The integral $\int_{t}^{\infty} f(s, x(s)) d s$ is convergent, and hence $T$ is well defined, as it fol- 
lows by considering $h_{\|x\|_{\infty}}$. Let $\epsilon=1+\sup _{a \leqslant t<+\infty} u(t)$ where $u$ is the maximal solution on $[a,+\infty[$ of

$$
u^{\prime}=\omega(t, u), \quad \lim _{t \uparrow \infty} u(t)=\left\|x_{\infty}\right\|+1 .
$$

Let $B$ be the closed ball in $X$ of center 0 and radius $\epsilon$. Using $h_{\epsilon}$ it is easily seen that $T_{m}(B)$ is an equicontinuous set, included at $+\infty$. Therefore, by Ascoli's theorem applied to $X, T_{m}(B)$ is a relatively compact set in $X$ for all $m$. Obviously $T_{m}$ is continuous and hence $\left.T_{m}\right|_{B}$ is a compact operator $B \rightarrow X$ for all $m$. Using $h_{\epsilon}$ it is easily seen that $\lim _{m} T_{m}=T$ uniformly in $B$. Therefore also $\left.T\right|_{B}$ is a compact operator $B \rightarrow X$. Obviously the solutions $x$ of the terminal value problem

$$
x^{\prime}=f(t, x), \quad \lim _{t \uparrow \infty} x(t)=x_{\infty},
$$

are characterized by the condition $x=T x$, i.e. the set of solutions of (1) coincides with the set $F(T)$ of fixed points of $T$. If $x \in F(T)$, then by

$$
\frac{d}{d t}\|x(t)\|^{2}=2\left(x^{\prime}(t) \mid x(t)\right)=2(f(t, x) \mid x) \geqslant 2 \omega(t,\|x(t)\|)\|x(t)\|
$$

and by the fact $u^{2}$ is the maximal solution in $[a,+\infty[$ of the terminal value problem

$$
w^{\prime}=g(t, w), \quad \lim _{t \uparrow \infty} w(t)=\left(\left\|x_{\infty}\right\|+1\right)^{2},
$$

with $g(t, w)=2 \omega(t, \sqrt{w}) \sqrt{w}(u \geqslant 0$ in view of the domain of $\omega)$ it follows from Theorem 1(ii) applied to $\|x(\cdot)\|^{2}, u^{2}$ and $g$ that

$$
\|x(t)\| \leqslant u(t) \quad(a \leqslant t<+\infty) .
$$

Therefore $F(T)$ is contained in the interior of $B$. Considering the intervals $[a+m-1 / m, a+m],[a+m-2 / m, a+m-1 / m]$, etc., from the definition of $T_{m}$ it is easily seen that $I-T_{m}, I$ the identity map of $X$, is a bijection $X \rightarrow$ $X$. This implies that $\left.\left(I-T_{m}\right)\right|_{B}$ is a homeomorphism into $B \rightarrow X$ (since $I-T_{m}$ is a proper map, hence closed) and that there is $x_{m}=T x_{m}$ for every $m$. Let

$$
\omega_{m}(t, x)=\omega(t+1 / m, x) \text { and } u_{m}(t)=u(t+1 / m) .
$$

If $x^{\prime}=\omega(t, x)$, then $x_{m}(t)=x(t+1 / m)$ is a solution of $x_{m}^{\prime}=\omega_{m}\left(t, x_{m}\right)$, and if $x_{m}^{\prime}=\omega_{m}\left(t, x_{m}\right)$ then $x(t)=x_{m}(t-1 / m)$ is a solution of $x^{\prime}=\omega(t, x)$. Therefore $u_{m}$ is the maximal solution of

$$
u_{m}^{\prime}=\omega_{m}\left(t, u_{m}\right), \quad \lim _{t^{\uparrow \infty}} u_{m}(t)=\left\|x_{\infty}\right\|+1
$$

Since $\lim _{t \uparrow \infty} u(t)=\left\|x_{\infty}\right\|+1$, there is $t_{0} \geqslant a$ such that 


$$
\left\|x_{\infty}\right\|<u_{m}(t) \quad\left(t \geqslant t_{0} ; m \in \mathbf{Z}^{+}\right) .
$$

Let $m_{0}$ be so large that $a+m_{0} \geqslant t_{0}$. Repeating the argument used to establish (2), from

$$
\frac{d}{d t}\left\|x_{m}(t)\right\|^{2} \geqslant 2 \omega_{m}\left(t,\left\|x_{m}(t)\right\|\right)\left\|x_{m}(t)\right\| \quad(a \leqslant t<m+a)
$$

and

$$
\left\|x_{m}(a+m)\right\|<u_{m}(a+m) \quad\left(m \geqslant m_{0}\right),
$$

we can deduce from Theorem 1(ii), applied to $\left\|x_{m}(\cdot)\right\|^{2}, u_{m}^{2}$ and $g_{m}(t, x)=$ $2 \omega_{m}(t, \sqrt{x}) \sqrt{x}$ in $[a, a+m[$ :

$$
\left\|x_{m}(t)\right\| \leqslant u_{m}(t)=u(t+1 / m) \quad\left(a \leqslant t<a+m ; m \geqslant m_{0}\right) .
$$

Thus $x_{m} \in B$ for all $m$. Then $F(T) \neq \varnothing$ as it follows easily from the uniform convergence of $\left(T_{m}\right)_{m}$ to $T$ in $B$ and the compactness of $\overline{T(B)}$. Moreover, since $F(T) \cap \partial B=\varnothing$ as seen above, Theorem 1.1 of Vidossich [9] applied to $I-T$ and $I-T_{m}$ shows that $F(T)$ is a compact connected subset of $X$. Q.E.D.

Repeating the argument in the proof of Corollary 1 to Theorem 4 we have the following result.

CorollaRy 1. Let $f:\left[a,+\infty\left[\times \mathbf{R}^{n} \rightarrow \mathbf{R}^{n}\right.\right.$ and $\omega:\left[a,+\infty\left[\times \mathbf{R}^{+} \rightarrow\right.\right.$ $\mathbf{R}^{+}$be continuous such that

$$
|(f(t, x) x)| \leqslant \omega(t,\|x\|)\|x\| \text { or }\|f(t, x)\| \leqslant \omega(t,\|x\|) .
$$

If for every $\epsilon>0$ there is $h_{\epsilon} \in L_{\mathrm{loc}}^{1}\left(\left[a,+\infty[)\right.\right.$ such that $\int_{a}^{+\infty} h_{\epsilon}<+\infty$ and

$$
\|f(t, x)\| \leqslant h_{\epsilon}(t) \quad(t \geqslant a ;\|x\| \leqslant \epsilon),
$$

and if for every $u_{\infty} \in \mathbf{R}^{+}$there is a maximal solution in $[a,+\infty[$ of

$$
u^{\prime}=-\omega(t, u), \quad \lim _{t \uparrow \infty} u(t)=u_{\infty},
$$

then for every $x_{\infty} \in \mathbf{R}^{n}$ there is a solution on $[a,+\infty$ [ of the terminal value problem

$$
x^{\prime}=f(t, x), \quad \lim _{t \uparrow \infty} x(t)=x_{\infty},
$$

and the set of solutions is connected and compact for the topology of uniform convergence on $[a,+\infty[$.

If $\omega(t, \cdot)$ is increasing, then there are cases in which we do not need the hypothesis concerning $h_{\epsilon} \in L^{1}([a,+\infty D$, as shown in the proof of the following result. The following corollary completes Theorem 5 of Brauer [1] since it shows the connectedness of the set of solutions. 
Corollary 2. Let $f:\left[a,+\infty\left[\times \mathbf{R}^{n} \rightarrow \mathbf{R}^{n}\right.\right.$ and $\omega:\left[a,+\infty\left[\times \mathbf{R}^{+} \rightarrow \mathbf{R}^{+}\right.\right.$ be continuous such that $\|f(t, x)\| \leqslant \omega(t,\|x\|)$. If $\omega(t, \cdot)$ is increasing for all $t$ and if the Cauchy problem

$$
u^{\prime}=\omega(t, u), \quad u\left(t_{0}\right)=u_{0},
$$

has a solution which is bounded in $\left[t_{0},+\infty\left[\right.\right.$ for all $t_{0}$ and $u_{0}$, then for every $x_{\infty} \in \mathbf{R}^{n}$ there is $a_{\infty} \in[a,+\infty[$ such that the terminal value problem

$$
x^{\prime}=f(t, x), \quad \lim _{t \uparrow \infty} x(t)=x_{\infty},
$$

has solutions in $\left[a_{\infty},+\infty[\right.$ and their set is compact and connected for the topology of uniform convergence on $\left[a_{\infty},+\infty[\right.$.

PRoof. Fix $x_{\infty} \in \mathbf{R}^{n}$ and $\epsilon>2\left\|x_{\infty}\right\|$. Let $r: \mathbf{R}^{n} \rightarrow\left\{x \in \mathbf{R}^{n} \mid\|x\| \leqslant \epsilon\right\}$ be the orthogonal projection and define $f_{\epsilon}:\left[a,+\infty\left[\times \mathbf{R}^{n} \rightarrow \mathbf{R}^{n}, g_{\epsilon}:[a,+\infty[\right.\right.$ $\times \mathbf{R} \rightarrow \mathbf{R}$ by

$$
\begin{aligned}
& f_{\epsilon}(t, x)=f(t, r(x)), \\
& g_{\epsilon}(t, u)= \begin{cases}\omega(t, \epsilon) & \text { if } u>\epsilon, \\
\omega(t, u) & \text { if } 0 \leqslant u \leqslant \epsilon, \\
\omega(t, 0) & \text { if } u<0\end{cases}
\end{aligned}
$$

Obviously $f_{\epsilon}, g_{\epsilon}$ are continuous, $g_{\epsilon}(t, \cdot)$ is increasing,

$$
\left\|f_{\epsilon}(t, x)\right\| \leqslant g_{\epsilon}(t,\|x\|), \quad\left|g_{\epsilon}(t, u)\right| \leqslant \omega(t,|u|),
$$

and $f_{\epsilon}(t, x)=f(t, x)$ for $\|x\| \leqslant \epsilon$. By the hypotheses of the corollary there exists a bounded solution $u_{\epsilon}$ of the Cauchy problem $u_{\epsilon}^{\prime}=\omega\left(t, u_{\epsilon}\right), u_{\epsilon}(a)=\epsilon$. Since $u_{\epsilon} \geqslant \epsilon$ and $u_{\epsilon}$ is increasing (by $\left.u_{\epsilon}^{\prime} \geqslant 0\right)$, since $\int_{a}^{t} \omega\left(s, u_{\epsilon}(s)\right) d s=u_{\epsilon}(t)-\epsilon$ and since $\omega(t, \cdot)$ is increasing, it is easily seen that the function $h_{\epsilon}=\omega\left(\cdot, u_{\epsilon}(\cdot)\right)$ has the following properties:

$$
\begin{aligned}
& \int_{a}^{+\infty} h_{\epsilon}<+\infty, \\
& \left.\|f(t, x)\| \leqslant h_{\epsilon}(t) \text { and }\left\|f_{\epsilon}(t, x)\right\| \leqslant h_{\epsilon}(t) \quad \text { (all } t ;\|x\| \leqslant \epsilon\right) \text {, } \\
& \left.\left\|g_{\epsilon}(t, u)\right\| \leqslant h_{\epsilon}(t) \quad \text { (all } t ;|u| \leqslant \epsilon\right) \text {. }
\end{aligned}
$$

Let $a_{\epsilon} \in\left[a,+\infty\left[\right.\right.$ be such that $\int_{a_{\epsilon}}^{\infty} h_{\epsilon}<\epsilon / 2$. By (1), Theorem 5 of Brauer [1] ensures that each terminal value problem

$$
\begin{array}{ll}
x^{\prime}=f(t, x), & \lim _{t \uparrow \infty} x(t)=x_{\infty}, \\
y^{\prime}=f_{\epsilon}(t, y), & \lim _{t \uparrow \infty} y(t)=x_{\infty},
\end{array}
$$




$$
u^{\prime}=-g_{\epsilon}(t, u), \quad \lim _{t \uparrow \infty} u(t)=\left\|x_{\infty}\right\|,
$$

has some solution in $\left[a_{\epsilon},+\infty[\right.$. We claim that the solution $x, y, u$ satisfy respectively

$$
\|x(\cdot)\| \leqslant \epsilon, \quad\|y(\cdot)\| \leqslant \epsilon, \quad|u(\cdot)| \leqslant \epsilon,
$$

in $\left[a_{\epsilon},+\infty[\right.$. We prove only the first inequality in (5), since the others have similar proof. Let

$$
\alpha=\inf \{t \in[a,+\infty[\mid\|x(\cdot)\| \leqslant \epsilon \text { in }[t,+\infty[\} .
$$

If $\alpha>a_{\epsilon}$, then by continuity we have $\|x(\alpha)\|=\epsilon$ and hence

$$
\begin{aligned}
\epsilon & =\|x(\alpha)\|=\left\|x_{\infty}-\int_{\alpha}^{+\infty} f(s, x(s)) d s\right\| \leqslant\left\|x_{\infty}\right\|+\int_{\alpha}^{+\infty}\|f(s, x(s))\| d s \\
& \leqslant\left\|x_{\infty}\right\|+\int_{\alpha}^{+\infty} h_{\epsilon}(s) d s \quad(\text { by }\|x(s)\| \leqslant \epsilon \text { for } s \geqslant \alpha) \\
& <\epsilon .
\end{aligned}
$$

Therefore $\alpha=a_{\epsilon}$, and the first inequality in (5) does hold. From (5) it follows:

(i) the set of solutions of (2) in $\left[a_{\epsilon},+\infty[\right.$ coincides with the set of solutions of (3) in $\left[a_{\epsilon},+\infty[\right.$; and

(ii) there exists a maximal solution of (4) in $\left[a_{\epsilon},+\infty[\right.$.

While (i) is clear from the definition of $f_{\epsilon}$, (ii) follows from the corollary to Theorem 1 of Vidossich [11]. Now we apply the above Corollary 1 of Theorem 5 to $f_{\epsilon}, g_{\epsilon}$ (note that $u \geqslant 0$ since $u$ is decreasing by $u^{\prime}=-g_{\epsilon}(t, u) \leqslant 0$, and hence we may restrict $g_{\epsilon}$ to $\left[a_{\epsilon},+\infty\left[\times \mathbf{R}^{+}\right.\right.$in order to apply Corollary 1) and we have that the set of solutions of (3), hence of (2), is connected and compact for the topology of uniform convergence on $\left[a_{\epsilon},+\infty[\right.$. Q.E.D.

5. Final remarks. (1) The argument used in the proof of Corollary 2 of Theorem 5 shows how to eliminate the hypotheses concerning uniqueness of Cauchy problem from Theorem 1 of Hallam [3] when $\omega(t, \cdot)$ is increasing.

(2) The results of $\$ 4$ can also be proved in a Banach space under a compactness assumption on $f$; but this was avoided for simplicity. We only note that in this way we can complete the existence theorem of Ladas and Lakshmikantham [6] by showing the connectedness of the set of solutions.

\section{REFERENCES}

1. F. Brauer, Global behavior of solutions of ordinary differential equations, J. Math. Anal. Appl. 2 (1961), 145-158. MR 24 \#A284.

2. F. Cafiero, Su un problema ai limiti relativo all'equazione $y^{\prime}=f(x, y, \lambda)$, Giorn. Mat. Battaglini (4) 77 (1947), 145-163. MR 10, 194.

3. T. G. Hallam, A comparison principle for terminal value problems in ordinary dif: ferential equations, Trans. Amer. Math. Soc. 169 (1972), 49-57. MR 46 \#5733. 
4. P. Hartman, Ordinary differential equations, Wiley, New York and London, 1964. MR 30 \#1270.

5. T. Kato, Nonlinear semigroups and evolution equations, J. Math. Soc. Japan 19 (1967), 508-520. MR 37 \#1820.

6. G. Ladas and V. Lakshmikantham, Global existence and asymptotic equilibrium in Banach spaces, J. Indian Math. Soc. 36 (1972), 33-40. MR 47 \#7169.

7. V. Lakshmikantham and S. Leela, Differential and integral inequalities. Vol. I, Academic Press, New York, 1969.

8. Ja. D. Mamedov, One-sided estimates in the conditions for existence and uniqueness of solutions of the limit Cauchy problem in a Banach space, Sibirsk. Mat. Ž 6 (1965), 11901196. (Russian) MR 32 \#908.

9. G. Vidossich, On Peano phenomenon, Boll. Un. Mat. Ital. (4) 3 (1970), 33-42. MR 42 \#6674.

10. Existence, comparison and asymptotic behavior of solutions of ordinary differential equations in finite and infinite dimensional Banach space, Notas de Matemática, no. 24, Universidade de Brasilia, 1972.

11. Two remarks on global solutions of ordinary differential equations in the real line, Proc. Amer. Math. Soc. 55 (1976), $111-115$.

DEPARTAMENTO DE MATEMÁTICA, UNIVERSIDADE DE BRASILIA, 70.000BRASÍLIA, D. F., BRAZIL

Current address: Istituto Matematico, Università di Trieste, 34127 Trieste, Italy 